\title{
Metabolomics and Transcriptomics of Metabolic Disorders
}

\author{
Laura Paternain • Javier Campion
}

Published online: 4 October 2013

(C) Springer Science+Business Media New York 2013

\begin{abstract}
The current prevalence of metabolic diseases such as obesity and type 2 diabetes mellitus (T2DM) is increasing not only due to genetic causes, but also because of lifestyle factors such as lack of physical exercise and consumption of high fat and high glycemic index diets, being one of the most serious public health problems around the world. Different omic approaches such as metabolomics or transcriptomics have revolutionized the quantitative analysis of an organism's state, linking cellular pathways to biological mechanisms, and could be applicable for identifying metabolic markers, which could be useful as diagnostic tools in a personalized healthcare setting. It may also open the door to novel biomarker discovery, disease diagnosis, and novel therapeutic avenues. This review covers the metabolomics and transcriptomic studies analyzing different biomarkers involved in the development of obesity and T2DM.
\end{abstract}

Keywords Obesity · Type 2 diabetes · Omics ·

Transcriptome $\cdot$ Metabolome

\section{Introduction}

Obesity, type 2 diabetes mellitus (T2DM), and metabolic syndrome are multifactorial diseases of considerable heterogeneity [1]. However, whereas obesity and T2DM diagnostic criteria are clearly defined, this is not the case for metabolic

L. Paternain · J. Campion $(\bowtie)$

Department of Nutrition, Food Science and Physiology, University of

Navarra, c/ Irunlarrea 1, 31008 Pamplona, Spain

e-mail: jcampion@unav.es

L. Paternain

e-mail: legatzpi@hotmail.es

J. Campion

CIBERobn, Physiopathology of Obesity and Nutrition, Institute of Health Carlos III, Madrid, Spain syndrome. Different definitions of metabolic syndrome have been proposed since 1998, when WHO defined this disease as a set of the presence of any type of diabetes mellitus, impaired glucose tolerance, impaired fasting glucose or insulin resistance, and two additional symptoms such as hypertension, dyslipidemia, central obesity and/or microalbuminuria [2]. Different organizations such as International Diabetes Federation, European Group for the Study of Insulin Resistance, and American Heart Association have also given a definition for metabolic syndrome, and although they differ in details, all agree on the essential components. Currently, the criteria represent a combination of metabolic perturbations including central obesity, insulin resistance, dyslipidemia characterized by high triglyceride and low high-density lipoprotein concentration, and hypertension.

The emerging epidemic of metabolic disorders around the world cannot be ignored. In 2010 more than 1.5 billion adults in the world were overweight, with nearly 500 million of them being obese $[3,4]$. Recent estimates predict there will be more than 400 million individuals with T2DM worldwide by the year 2030 [5]. Moreover, despite the differences between definitions, U.S. estimates show that the prevalence of metabolic syndrome among adults ranges form $34.3 \%$ to $38.5 \%$ depending on the criteria used [6]. Sedentary lifestyles and the high intake of foods rich in fat and sugar have raised the prevalence of these pathologies, making them the most serious public health problems around the world [7].

Due to the increasing prevalence of metabolic diseases, the identification of molecular biomarkers for assessing the risk of obesity, T2DM, and metabolic syndrome would offer a better, non-invasive way to predict patient risk and to prevent metabolic diseases in humans. Molecular biomarkers have been defined as biomarkers that can be discovered using basic and acceptable platforms such as genomics and metabolomics. This review covers the transcriptomics and metabolomics studies analyzing different biomarkers involved in the development of metabolic diseases such as obesity and T2DM. 
Thus, identifying metabolic markers and pathways of diseaseassociated intermediate phenotypes is very important, as these biomarkers could be applicable as diagnostic tools in a personalized healthcare setting and may also open the door to novel biomarker discovery, disease diagnosis, and novel therapeutic avenues.

\section{Metabolomics technologies}

Metabolomics is a systematic analysis of low molecularweight biochemical compounds in a biological cell, tissue, organ, or organism, and it has been increasingly applied to discovering biomarkers, identifying perturbed pathways, diagnosing diseases, and measuring the response to treatment [8]. Metabolic profiling can give an instantaneous snapshot of the physiology of the organism and can be seen as the gap between genotype and phenotype [4]. Moreover, metabolomics brings the opportunity to discover new biomarkers important for identifying and stratifying diseases, predicting their progression, and determining the effectiveness, safety and dosage of therapeutic interventions [4].

Nuclear magnetic resonance (NMR) spectroscopy and mass spectroscopy (MS) are the two most used highthroughput tools in metabolomics studies, and both can provide complementary snapshots of the metabolome of body fluids [9]. However, the combination of both techniques has the advantage of complementary analytical outcomes and validation of the results obtained separately.

Biomarkers and metabolomics studies in metabolic disorders

\section{Obesity}

Obesity is a disorder of the whole body involving metabolic changes, but the actual alterations in metabolism during obesity and any dysfunction associated with this disease at the level of individual organs or cellular organelles are not yet clearly understood [10]. In recent years different studies have been published identifying many obesity-related metabolites implied in several pathways clarifying the metabolic intricacies of obesity (Table 1).

Glucose is the most important source of energy for most organisms, and it is known that glucose metabolism is altered in obesity. Thus, higher blood lactate levels in obese humans have been found [11]. Lactate is also the major precursor for gluconeogenesis in the body, and the rise in plasma lactate in obesity may be an indicator of perturbation of hepatic glucose production and hepatic lipid synthesis. Thus, lactate is an important obesity-related metabolite identified by metabolomics [12•].

Lipids are another important source and store of energy for metabolism, and different studies have observed changes in lipid metabolism due to obesity. Pietiläinen et al. [13] used
Ultraperformance liquid chromatography/Mass spectrometry (UPLC/MS) technology to investigate whether acquired obesity was associated with changes in global serum lipid profiles independent of genetic factors in young adult monozygotic twins. This study revealed an increase in lysophosphatidylcholines (lysoPCs) and a decrease in diethyl ether phospholipids related to obesity, independent of genetic influence. According to these results and with the same technology, Kim et al. [14] identified three lysoPCs in plasma as potential obesity biomarkers comparing overweight/obese and normal-weight men. Especially, overweight/obese subjects showed higher levels of lysoPC C14:0 and lysoPC C16:0, and lower levels of lysoPC C18:1. Obesity is also associated with elevated levels of free fatty acids (FFAs) [15] in the bloodstream; compared to conventional targeted analysis, metabolomics can provide a more unbiased model of the relationship of FFAs to other small molecules, which may help to understand obesity. For example, higher levels of stearic acid, total saturated fatty acids, palmitoleic acid, and lower linoleic acid levels, as well as the variation of amino acids and carnitine in plasma, were detected in overweight/obese men comparing to normal-weight men [14]. In a more recent study, Oberbach et al. [16] showed that obese individuals had higher levels of glycerolPC 42:0 and lower levels of diacylglycerolPC (diacylglycerolPC 32:0, 32:1 and 40:5) with MS/MS technology.

The branched chain amino acids (BCAAs) leucine, isoleucine, and valine are among the nine essential amino acids for humans. The BCAAs play important roles in protein synthesis [17], improve glucose metabolism and oxidation [18], and regulate food intake and the leptin secretion from body fat [19]. Similar to the Oberbach study, Newgard et al. [11] observed differences in the blood amino acid profiles of obese subjects compared with lean subjects. Through metabolic analysis they showed higher levels of a combination of BCAA (leucine/isoleucine and valine), glutamate/glutamine, and the aromatic amino acids phenylalanine and tyrosine in obese individuals than in lean individuals.

\section{Type 2 diabetes mellitus}

T2DM is a chronic disease characterized by high blood glucose levels, which may be due to the development of insulin resistance and subsequently the loss of $\beta$-cell function. T2DM is related to lifestyle factors including diet, insufficient physical activity, an overweight or obese state, and stress. Whereas the diagnosis and treatment of T2DM have been thoroughly investigated, the identification of novel pathways or early biomarkers related to the development of the disease is still underway.

Comparisons between the levels of various metabolites in diabetic subjects and healthy subjects revealed changes involving different metabolic pathways, as seen in the previous 
Table 1 Differentially regulated metabolites in some metabolic disorders
${ }^{1}(+)$ and $(-)$ indicate an increase or decrease in relative metabolite concentration, respectively, when comparing metabolic disease with control

PC, Phosphatidylcholine; BCAA; Branch chained amino acids; MS/ MS, mass tandem spectrometry; ULPC, ultraperformance liquid chromatography; UHPLC, Ultrahighperformance liquid chromatography; GC, Gas chromatography

\begin{tabular}{|c|c|c|c|c|c|}
\hline $\begin{array}{l}\text { Metabolic } \\
\text { disorder }\end{array}$ & Pathway & Metabolite $^{1}$ & Sample & Analytical platform & Reference \\
\hline \multirow[t]{19}{*}{ Obesity } & $\begin{array}{l}\text { Glucose } \\
\text { metabolism }\end{array}$ & Lactate $(+)$ & Serum & MS/MS & {$[1]$} \\
\hline & \multirow[t]{11}{*}{$\begin{array}{l}\text { Lipid } \\
\quad \text { metabolism }\end{array}$} & $\begin{array}{l}\text { LysoPCs }(+) \\
\text { Diethyl ether phospholipids (-) }\end{array}$ & Serum & UPLC/MS & {$[2]$} \\
\hline & & $\begin{array}{l}\text { LysoPC 14:0 }(+) \\
\text { LysoPC 16:0 (+) }\end{array}$ & Plasma & $\begin{array}{l}\text { UPLC/Q-TOF } \\
\text { MS }\end{array}$ & {$[3]$} \\
\hline & & LysoPC 18:1 (-) & & & \\
\hline & & Stearic acid $(+)$ & & & \\
\hline & & Saturated fatty acids $(+)$ & & & \\
\hline & & Palmitoleic acid $(+)$ & & & \\
\hline & & Linoleic acid (-) & & & \\
\hline & & Carnitine (-) & & & \\
\hline & & $\begin{array}{l}\text { GlycerolPC 42:0 (+) } \\
\text { DiacylglycerolPC 32:0 (-) }\end{array}$ & Serum & MS/MS & {$[4]$} \\
\hline & & DiacylglycerolPC 32:1 (-) & & & \\
\hline & & DiacylglycerolPC 40:5 (-) & & & \\
\hline & \multirow{7}{*}{$\begin{array}{l}\text { Amino acid } \\
\quad \text { metabolism }\end{array}$} & $\operatorname{BCAA}(+)$ & Serum & MS/MS & {$[1]$} \\
\hline & & & Plasma & UPLC/Q-TOF MS & {$[3]$} \\
\hline & & Phenylalanine $(+)$ & Serum & MS/MS & {$[1]$} \\
\hline & & & Plasma & UPLC/Q-TOF MS & {$[3]$} \\
\hline & & Tryptophan (+) & Serum & MS/MS & {$[1]$} \\
\hline & & & Plasma & UPLC/Q-TOF MS & {$[3]$} \\
\hline & & Glutamate/glutamine $(+)$ & Serum & MS/MS & {$[1]$} \\
\hline \multirow[t]{11}{*}{ Diabetes } & \multirow[t]{3}{*}{$\begin{array}{l}\text { Glucose } \\
\quad \text { metabolism }\end{array}$} & $\begin{array}{l}\text { Glucose }(+) \\
\text { Acetone }(+)\end{array}$ & Plasma & $\begin{array}{l}\mathrm{UHPLC} / \mathrm{MS} / \mathrm{MS} 2 \\
\text { and } \mathrm{GC} / \mathrm{MS}\end{array}$ & {$[5]$} \\
\hline & & Acetoacetic acid $(+)$ & & & \\
\hline & & $\beta$-hydroxybutyrate $(+)$ & & & \\
\hline & \multirow[t]{4}{*}{$\begin{array}{l}\text { Lipid } \\
\quad \text { metabolism }\end{array}$} & $\begin{array}{l}\text { Phosphatidylethanolamines }(+) \\
\text { Arachidonate }(-)\end{array}$ & Plasma & $\begin{array}{l}\mathrm{UHPLC} / \mathrm{MS} / \mathrm{MS} 2 \\
\text { and } \mathrm{GC} / \mathrm{MS}\end{array}$ & {$[5]$} \\
\hline & & Palmitate $(+)$ & & & \\
\hline & & Linoleate $(+)$ & & & \\
\hline & & Acylcarnitines $(+)$ & Plasma & MS/MS & {$[6]$} \\
\hline & \multirow[t]{4}{*}{$\begin{array}{l}\text { Amino acid } \\
\quad \text { metabolism }\end{array}$} & $\begin{array}{l}\text { BCAA }(+) \\
\text { Phenylalanine }(+)\end{array}$ & \multirow[t]{4}{*}{ Plasma } & \multirow{4}{*}{$\begin{array}{l}\text { MS/MS, UHPLC/ } \\
\text { MS/MS2 and } \\
\text { GC/MS }\end{array}$} & \multirow[t]{4}{*}[5,7]{} \\
\hline & & Tyrosine $(+)$ & & & \\
\hline & & Alanine $(+)$ & & & \\
\hline & & Tryptophan $(+)$ & & & \\
\hline
\end{tabular}

section (Table 1). As expected, diabetic individuals exhibited differences in glucose metabolism, showing higher levels of glucose or mannose levels [20]. Moreover, higher levels of acetone, acetoacetate, and $\beta$-hydroxybutyrate were observed in T2DM patients [20], indicating ketoacidotic metabolic decompensation.

Alterations in fatty acid metabolism have been extensively studied in patients with diabetes, using different metabolomics technologies such as ultra-high performance liquid chromatography/tandem mass spectrometry (UHPLC/ $\mathrm{MS} / \mathrm{MS} 2$ ) or/and gas chromatography/mass spectrometry
(GC/MS). Thus, increased fatty acid levels were observed in T2DM individuals [20] as well as in patients with impaired glucose tolerance [21•]. Shure et al. [20] showed that arachidonate levels are, on average, lower in the diabetes group, while levels of long-chain fatty acids such as linoleate and palmitate are higher. Moreover, Mihalik et al. [22] detailed almost 20 different fatty acids including saturated, monounsaturated, and polyunsaturated fatty acids affected by T2DM, showing higher fasting levels of long-chain saturated and monounsaturated acylcarnitines in T2DM subjects compared with lean controls. It is known that 
elevated levels of FFA induce insulin resistance in muscle and liver tissue by decreasing insulin-stimulated glucose uptake and glycogenesis [23]. Although the underlying mechanism is not completely understood, it has been hypothesized that it is related to oxidative stress or inflammatory lipid pathways [15].

As was previously observed in the Obesity section, a broad range of amino acids were also affected in diabetic patients, including the BCAA, phenylalanine, tyrosine, alanine, and tryptophan, which occur at higher levels in T2DM individuals in comparison with control subjects [20]. Similarly, a study of Asian and Chinese men demonstrated that perturbations in amino acid metabolism, including BCAA, were associated with insulin resistance [24].

Although a detailed understanding of the pathophysiology of T2DM is important, the identification of early metabolic alterations is a prerequisite for the development of preventive strategies. Thus, there are different studies that attempt to address this issue. Several biomarkers have been proposed as indicators for T2DM risk, such as fasting plasma glucose levels [25] or inflammatory markers [26], but most of them do not realize the complexity of this disease [27]. However, the predictive ability of amino acid levels, including BCAA, was revealed in different studies.

Wang et al. [28•] followed normoglycemic individuals for 12 years in the Framingham Offspring Study; 201 subjects developed diabetes. They observed that fasting plasma concentration of BCAA, tyrosine, and phenylalanine were elevated 12 years before the onset of T2DM and were also linked to a higher diabetes risk. In fact, a combination of three of the amino acids predicted future diabetes with a more than 5-fold higher risk for individuals in the top quartile of the amino acid concentration levels.

Wang-Sattler et al. [29•] also identified novel biomarkers for pre-diabetes in a recently published work. They quantified 140 metabolites in fasting serum samples of 4,297 individuals from the population-based Cooperative Health Research in the Region of Augsburg (KORA) cohort. In this study they determined that glycine, lysoPC (18:2), and acetylcarnitine, which are impaired glucose tolerance-specific metabolites, are significantly related to the pre-diabetes state, showing that low levels of glycine and lysoPC at baseline predict the risk of developing T2DM, even seven years before the onset of the disease. Moreover, those two metabolites were replicated in the European Prospective Investigation into Cancer and Nutrition (EPIC)-Potsdam cross-sectional study published in 2013 [30].

Metabolomics has revolutionized the quantitative analysis of small molecules, not only linking cellular pathways to biological mechanisms, but also for elucidating the snapshot of human health and/or disease, which could be used to yield molecular targets for therapeutic intervention in a noninvasive manner.
Transcriptomics methodology

The transcriptome is the set of all RNA molecules produced in one or a population of cells, and their quantity, for a specific developmental stage or physiological condition. Understanding the transcriptome is as essential as recognizing the metabolites discussed previously for interpreting the functional elements of the genome, revealing the molecular constituents of cells and tissues, and also for understanding development and disease [31].

The aims of transcriptomics are to catalogue different species of transcripts, to determine the transcriptional structure of the genes, and to quantify the expression levels of each transcripts during development and under different conditions [31]. In this review, we will focus on the changes in expression levels of mRNA in metabolic disorders.

Different technologies have been developed to deduce and quantify the transcriptome including hybridization or sequencebased approaches. However these techniques, although being the most affordable, have many limitations. Recently developed techniques provide novel high-throughput DNA sequencing methods for both mapping and quantifying transcriptomes. This method, termed RNA-Seq (RNA sequencing), has clear advantages over existing approaches and has already been applied to different organisms such as Saccharomyces cerevisiae, Arabidopsis thaliana, and mouse and human cells [32-34].

Transcriptomic studies of metabolic disorders

\section{Obesity}

There is a huge amount of evidence for the genetic basis of obesity studied in different human and animal models [35]. Different independent studies and meta-analyses of existing data from genome-wide association studies have implicated a total of 18 genetic loci as relevant for body weight regulation to date [36]. Moreover, apart from DNA sequence variants, genetic influences are also manifested through differences in gene expression levels in different relevant tissues; the blood transcriptome has also been found to be a valid non-invasive biological readout to generate information of high predictive and diagnostic content [37•] (Table 2).

Ghosh et al. [37•] carried out a whole-genome expression profiling of whole blood from 17 obese subjects (body mass index (BMI) of $30-50 \mathrm{~kg} / \mathrm{m}^{2}$ ) enrolled in the Ottawa Hospital Weight Management Program at the Ottawa Hospital, and 17 well matched lean subjects from the same genetic ancestry (Northern European White), with a BMI $\leq$ the 10th percentile for age and sex and no prior history of having had a BMI> 25 th percentile for more than a 2-year consecutive period. Using Affymetrix GeneChip ${ }^{\circledR}$ Human Genome U133 Plus 2.0 Arrays, they detected 12,127 probe sets, among which 374 probe sets were overexpressed (2-fold or greater), and 75 
Table 2 Transcriptomic studies in some metabolic disorders

\begin{tabular}{|c|c|c|c|c|c|}
\hline $\begin{array}{l}\text { Metabolic } \\
\text { disorder }\end{array}$ & Metabolic Pathway & Transcripts & Sample & Analytical platform & Reference \\
\hline \multirow[t]{17}{*}{ Obesity } & \multirow[t]{3}{*}{ Apoptosis } & Caspase 7 & \multirow[t]{13}{*}{$\begin{array}{l}\text { Whole } \\
\text { blood }\end{array}$} & $\begin{array}{l}\text { Affymetrix GeneChip }{ }^{\circledR} \\
\text { Human Genome } \\
\text { U133 Plus } 2.0 \\
\text { Arrays }\end{array}$ & {$[1]$} \\
\hline & & Fas & & & {$[1]$} \\
\hline & & Interleukin $1-\beta$ & & & {$[1]$} \\
\hline & \multirow[t]{5}{*}{ Ribosome } & RP L31 & & & {$[1]$} \\
\hline & & RP S7 & & & {$[1]$} \\
\hline & & RP S24 & & & {$[1]$} \\
\hline & & RP L35 & & & {$[1]$} \\
\hline & & RP L7 & & & {$[1]$} \\
\hline & \multirow{5}{*}{$\begin{array}{l}\text { Oxidative } \\
\text { phosphorylation }\end{array}$} & $\mathrm{COX} 6 \mathrm{C}$ & & & {$[1]$} \\
\hline & & COX7B & & & {$[1]$} \\
\hline & & $\mathrm{COX} 7 \mathrm{C}$ & & & {$[1]$} \\
\hline & & $\begin{array}{l}\text { NADH } \\
\text { coenzyme Q } \\
\text { reductase }\end{array}$ & & & {$[1]$} \\
\hline & & NDUFB3 & & & {$[1]$} \\
\hline & \multirow[t]{4}{*}{ Cell cycle } & BMAL1 & \multirow[t]{4}{*}{ PBMC } & \multirow[t]{4}{*}{ RT-PCR } & {$[2]$} \\
\hline & & CRY 1 & & & {$[2]$} \\
\hline & & CRY 2 & & & {$[2]$} \\
\hline & & PER 2 & & & {$[2]$} \\
\hline \multirow[t]{6}{*}{ Diabetes } & \multirow[t]{2}{*}{ Lipid metabolism } & ABCA1 & \multirow[t]{2}{*}{ PBMC } & \multirow[t]{2}{*}{$\begin{array}{l}\text { Whole human } \\
\text { genome oligo- } \\
\text { microarray }\end{array}$} & {$[3]$} \\
\hline & & GOS2 & & & {$[3]$} \\
\hline & \multirow[t]{2}{*}{$\begin{array}{l}\text { Nucleobase Nucleoside } \\
\text { Nucleotide and } \\
\text { Nucleic Acid } \\
\text { Metabolic Process }\end{array}$} & ILF2 & \multirow[t]{4}{*}{$\begin{array}{l}\text { Peripheral } \\
\text { blood }\end{array}$} & \multirow[t]{4}{*}{$\begin{array}{l}\text { Human exonic } \\
\text { evidence-based } \\
\text { oligonucleotide } \\
\text { array }\end{array}$} & {$[4]$} \\
\hline & & NFATC4 & & & {$[4]$} \\
\hline & \multirow[t]{2}{*}{ Signal Transduction } & MAPK11 & & & {$[4]$} \\
\hline & & GPX1 & & & {$[4]$} \\
\hline
\end{tabular}

RP, Ribosome protein; COX, Cytochrome coxidase; NDUFB3, NADH deyhdrogenase beta subcomplex 3; BMAL1, Brain and muscle Arnt-like protein-1; CRY, Cryptochrome; PER, Period; ABCA1, ATP-binding cassette sub-family A transporter; GOS2, G0/G1 switch 2; ILF2, nuclear factor of activated cells; NFATC4, nuclear factor of activated T-cells, cytoplasmic, calcineurin-dependent 1 ; MAPK11, mitogen-activated protein kinase 11; GPX1, glutathione peroxidase 1 probe sets were underexpressed (2-fold or greater) in the obese samples compared to the leans. These results were subjected to bioinformatics, and the pathway analysis was conducted with Kyoto Encyclopedia for Genes and Genomes (KEGG) metabolic pathway database identifying the 'apoptosis', 'ribosome', and 'oxidative phosphorylation' pathways. Different genes such as Caspase 7, Fas, or Interleukin $1-\beta$ related to apoptosis pathway, and/or ribosomal protein (RP) L31, S7, S24, L35, or L7 genes related to ribosome pathway were upregulated in obese subjects. Several genes involved in the mitochondrial process of electron transfer and ATP synthesis such as cytochrome c oxidase (COX) subunits 6C, 7B and 7C, NADH coenzyme $\mathrm{Q}$ reductase, and NADH dehydrogenase beta subcomplex 3 (NDUFB3) demonstrated increased expression in the obese cohort, leading to a significant enrichment of the 'oxidative phosphorylation' pathway in this group.

It is known that adipose tissue, being responsible for the storage of excess energy derived from the diet as fat, secretes a number of adipokines, which clearly exhibit daily variation in animal models and humans [38, 39]. Thus, plasma adiponectin concentrations in non-obese subjects show a $24 \mathrm{~h}$ rhythmic cycle, which is absent in obese subjects $[40,41]$. In this context, Tahira et al. [42] investigated the association between daytime variations in gene expression related to circadian rhythm and obesity, testing peripheral blood mononuclear cells by Real-time quantitative PCR. They showed that the expression levels of clock genes such as brain and muscle Arnt-like protein-1 (BMAL1), Cryptochrome (CRY) 1 and 2, and Period (PER) 2 were significantly higher during the day compared to those in 
the non-obese group. In addition, expression of BMAL1, CRY1, CRY2, and PER2 genes in blood cells increased between 12:00 and 21:00 in the obese group, suggesting a contribution of the chronotypes to fat accumulation in humans.

\section{Type 2 diabetes mellitus}

Many clinical and epidemiological studies have been carried out to elucidate the causalities of T2DM [43, 44]. However, previous studies focused on gene expression in T2DM have been restricted to adipose tissue and muscle or to animal models with limited sample size [45•]. Considering this review's objective of analyzing different biomarkers in a noninvasive way and the difficulty of obtaining permission to use human tissue samples such as liver, adipose, or muscle, the peripheral blood could be a convenient sample source for genetic studies [45•] (Table 2).

Mao et al. [45 ${ }^{\circ}$ performed a whole human genome oligomicroarray of peripheral white blood cells from 60 healthy and 84 T2DM subjects and found 30 differentially expressed genes in T2DM patients in comparison with healthy controls. They identified ATP-binding cassette sub-family A transporter (ABCA1) and G0/G1 switch 2 (GOS2), which are reported to be associated with lipid metabolism and T2DM in gene knockout cell lines and/or animal models [46-48], as new genetic markers in human for diagnosis and pharmaceutical drug design.

Previously, Grayson et al. [49] performed a transcriptomic study on peripheral blood cells of type 2 diabetic patients, using Human Exonic Evidence-Based Oligonucleotide array. After the array analysis with Gene Ontology, they reported an increased expression of genes associated with activation, signaling, and function of $\mathrm{T}$ cells. One of the sets, named Nucleobase Nucleoside Nucleotide and Nucleic Acid Metabolic Process, included the overexpression of the nuclear factor of activated cells (ILF2), which modulates IL-2 expression, and the nuclear factor of activated T-cells and cytoplasmic calcineurin-dependent 1 (NFATC4), a gene involved in the inducible expression of cytokines. The Signal Transduction gene set includes the mitogen-activated protein kinase 11 gene, MAPK11, which encodes a protein activated by pro-inflammatory cytokines and is over-expressed in this gene set, along with glutathione peroxidase 1 (GPX1). These results reflected the possibility that components of the adaptive immune system may contribute to the pathogenesis of T2D.

Transcriptomic analysis is improving constantly with new technologies, better suited analysis and data interpretation tools, and by its high level of customization and automation. However, there are still technical and biological problems to be solved before changes in mRNA levels can be taken as predictive for human health or disease.

\section{Concluding remarks}

Currently the incidence of metabolic diseases worldwide is alarming. Sedentary lifestyles and the high intake of foods rich in fat and sugars have increased the prevalence of metabolic diseases. In this context, the identification of molecular biomarkers for assessing the risk of obesity, T2DM, and metabolic syndrome is crucial to predict risk and prevent metabolic diseases in humans. This review summarizes different metabolomics and transcriptomic studies focused especially on obesity and T2DM.

Metabolomics has revolutionized metabolic disease research, linking cellular pathways to biological mechanisms and providing a global picture of the organism. From a technology perspective, metabolomics has come along at precisely the right time [50]; however, the different technologies used (MS and NMR spectroscopy) to gather metabolomics data have specific advantages and disadvantages when conducting metabolomic studies. The main advantage of MS is sensitivity, but one of the major weaknesses of MS in metabolomics is the quantification. Moreover, the major weaknesses of MS are the major strengths of NMR spectroscopy. However, the combination of both techniques has the advantage of complementary analytical outcomes and validation of the results obtained separately.

Understanding the transcriptome is essential for interpreting the functional elements of the genome and revealing the molecular constituents of cells and tissues, and also for understanding development and disease [31]. However, different studies have shown that the correlation between mRNA and protein expression, which exerts the effect on the organism, can be low due to post-transcription machinery [51]. In this context, the study of metabolomics is presently considered more realistic and easier to validate and use as a biomarker analysis than transcriptomic analysis. Thus, the metabolic profile exhibits the real surrogate endpoint of obesity and T2DM and seems to be a better approach to show the global physiology snapshot.

However, in order to deep in disease biomarkers research, an integral approach of different omics could be of interest. Thus, combining metabolomic and transcriptomic data by bioinformatics approaches could show not only the obvious effect of the mRNA levels on the metabolites present in the blood, but also the effects of these metabolites on different gene expression levels in the context of a metabolic disease.

\section{Compliance with Ethics Guidelines}

Conflict of Interest Laura Paternain and Javier Campion declare that they have no conflict of interest.

Human and Animal Rights and Informed Consent This article does not contain any studies with human or animal subjects performed by any of the authors. 


\section{References}

Papers of particular interest, published recently, have been highlighted as:

- Of importance

1. Zimmet P, Alberti KG, Shaw J. Global and societal implications of the diabetes epidemic. Nature. 2001;414(6865):782-7. doi:10.1038/414782a.

2. Alberti KG, Zimmet PZ. Definition, diagnosis and classification of diabetes mellitus and its complications. Part 1: diagnosis and classification of diabetes mellitus provisional report of a WHO consultation. Diabet Med. 1998;15(7):539-53.

3. (WHO) WHO. Global strategy on diet, physical activity and health. 2011. https://http://www.who.int/dietphysicalactivity/childhood/en/.

4. Zhang A, Sun H, Wang X. Power of metabolomics in biomarker discovery and mining mechanisms of obesity. Obes Rev. 2012. doi: 10.1111/obr.12011.

5. Shaw JE, Sicree RA, Zimmet PZ. Global estimates of the prevalence of diabetes for 2010 and 2030. Diabetes Res Clin Pract. 2010;87(1): 4-14. doi:10.1016/j.diabres.2009.10.007.

6. Ford ES, Li C, Zhao G. Prevalence and correlates of metabolic syndrome based on a harmonious definition among adults in the US. J Diabetes. 2010;2(3):180-93. doi:10.1111/ j.1753-0407.2010.00078.x.

7. Marti A, Martinez-Gonzalez MA, Martinez JA. Interaction between genes and lifestyle factors on obesity. Proc Nutr Soc. 2008;67(1):1-8. doi:10.1017/S002966510800596X.

8. Zhang A, Sun H, Wu X, Wang X. Urine metabolomics. Clin Chim Acta. 2012;414:65-9. doi:10.1016/j.cca.2012.08.016.

9. Zhang A, Sun H, Wang X. Serum metabolomics as a novel diagnostic approach for disease: a systematic review. Anal Bioanal Chem. 2012;404(4):1239-45. doi:10.1007/s00216-012-6117-1.

10. Kussmann M, Raymond F, Affolter M. OMICS-driven biomarker discovery in nutrition and health. J Biotechnol. 2006;124(4):758-87. doi:10.1016/j.jbiotec.2006.02.014.

11. Newgard CB, An J, Bain JR, Muehlbauer MJ, Stevens RD, Lien LF, et al. A branched-chain amino acid-related metabolic signature that differentiates obese and lean humans and contributes to insulin resistance. Cell Metab. 2009;9(4):311-26. doi:10.1016/j.cmet.2009.02.002.

12. - Xie B, Waters MJ, Schirra HJ. Investigating potential mechanisms of obesity by metabolomics. J Biomed Biotechnol. 2012;2012: 805683. doi:10.1155/2012/805683. This article focused on different obesity-related individual metabolites identified with metabolomics.

13. Pietilainen KH, Sysi-Aho M, Rissanen A, Seppanen-Laakso T, Yki-Jarvinen H, Kaprio J, et al. Acquired obesity is associated with changes in the serum lipidomic profile independent of genetic effects-a monozygotic twin study. PLoS One. 2007;2(2):e218. doi:10. 1371/journal.pone.0000218.

14. Kim JY, Park JY, Kim OY, Ham BM, Kim HJ, Kwon DY, et al. Metabolic profiling of plasma in overweight/obese and lean men using ultra performance liquid chromatography and Q-TOF mass spectrometry (UPLC-Q-TOF MS). J Proteome Res. 2010;9(9): 4368-75. doi:10.1021/pr100101p.

15. Boden G. Obesity, insulin resistance and free fatty acids. Curr Opin Endocrinol Diabetes Obes. 2011;18(2):139-43. doi:10.1097/MED. 0b013e3283444b09.

16. Oberbach A, Bluher M, Wirth H, Till H, Kovacs P, Kullnick Y, et al. Combined proteomic and metabolomic profiling of serum reveals association of the complement system with obesity and identifies novel markers of body fat mass changes. J Proteome Res. 2011;10(10):4769-88. doi:10.1021/pr2005555.
17. Blomstrand E, Eliasson J, Karlsson HK, Kohnke R. Branched-chain amino acids activate key enzymes in protein synthesis after physical exercise. J Nutr. 2006;136(1 Suppl):269S-73S.

18. Doi M, Yamaoka I, Nakayama M, Sugahara K, Yoshizawa F. Hypoglycemic effect of isoleucine involves increased muscle glucose uptake and whole body glucose oxidation and decreased hepatic gluconeogenesis. Am J Physiol Endocrinol Metab. 2007;292(6): E1683-93. doi:10.1152/ajpendo.00609.2006.

19. Lynch CJ, Gern B, Lloyd C, Hutson SM, Eicher R, Vary TC. Leucine in food mediates some of the postprandial rise in plasma leptin concentrations. Am J Physiol Endocrinol Metab. 2006;291(3): E621-30. doi:10.1152/ajpendo.00462.2005.

20. Suhre K, Meisinger C, Doring A, Altmaier E, Belcredi P, Gieger C, et al. Metabolic footprint of diabetes: a multiplatform metabolomics study in an epidemiological setting. PLoS One. 2010;5(11):e13953. doi:10.1371/journal.pone.0013953.

21. - Zhao X, Fritsche J, Wang J, Chen J, Rittig K, Schmitt-Kopplin P, et al. Metabolomic fingerprints of fasting plasma and spot urine reveal human pre-diabetic metabolic traits. Metabolomics. 2010;6(3):362-74. doi:10.1007/s11306-010-0203-1. This article shows novel insights into the metabolic alterations occurring in the lon, asymptomatic period preceding the manifestation of T2DM, giving prospects for new intervention targets.

22. Mihalik SJ, Goodpaster BH, Kelley DE, Chace DH, Vockley J, Toledo FG, et al. Increased levels of plasma acylcarnitines in obesity and type 2 diabetes and identification of a marker of glucolipotoxicity. Obesity (Silver Spring). 2010;18(9):1695700. doi:10.1038/oby.2009.510.

23. Wilding JP. The importance of free fatty acids in the development of Type 2 diabetes. Diabet Med. 2007;24(9):934-45. doi:10.1111/j. 1464-5491.2007.02186.x.

24. Tai ES, Tan ML, Stevens RD, Low YL, Muehlbauer MJ, Goh DL, et al. Insulin resistance is associated with a metabolic profile of altered protein metabolism in Chinese and Asian-Indian men. Diabetologia. 2010;53(4):757-67. doi:10.1007/s00125-009-1637-8.

25. Peters AL, Davidson MB, Schriger DL, Hasselblad V. A clinical approach for the diagnosis of diabetes mellitus: an analysis using glycosylated hemoglobin levels. Meta-analysis Research Group on the Diagnosis of Diabetes Using Glycated Hemoglobin Levels. Jama. 1996;276(15):1246-52.

26. Swellam M, Sayed M, Abdel-Fatah AA. Clinical implications of adiponectin and inflammatory biomarkers in type 2 diabetes mellitus. Dis Markers. 2009;27(6):269-78. doi:10.3233/DMA2009-0672.

27. Herder C, Baumert J, Zierer A, Roden M, Meisinger C, Karakas M, et al. Immunological and cardiometabolic risk factors in the prediction of type 2 diabetes and coronary events: MONICA/KORA Augsburg case-cohort study. PLoS One. 2011;6(6):e19852. doi:10.1371/ journal.pone.0019852.

28. - Wang TJ, Larson MG, Vasan RS, Cheng S, Rhee EP, McCabe E, et al. Metabolite profiles and the risk of developing diabetes. Nat Med. 2011;17(4):448-53. doi:10.1038/nm.2307. In this article the authors identified metabolites whose fasting levels at a routine examination could predict the future development of diabetes in healthy, normoglycemic individuals.

29. - Wang-Sattler R, Yu Z, Herder C, Messias AC, Floegel A, He Y, et al. Novel biomarkers for pre-diabetes identified by metabolomics. Mol Syst Biol. 2012;8:615. doi:10.1038/msb.2012.43. In this article the authors identify candidate biomarkers of pre-diabetes.

30. Floegel A, Stefan N, Yu Z, Muhlenbruch K, Drogan D, Joost HG, et al. Identification of serum metabolites associated with risk of type 2 diabetes using a targeted metabolomic approach. Diabetes. 2013;62(2):639-48. doi:10.2337/db12-0495.

31. Wang Z, Gerstein M, Snyder M. RNA-Seq: a revolutionary tool for transcriptomics. Nat Rev Genet. 2009;10(1):57-63. doi:10. $1038 / \mathrm{nrg} 2484$. 
32. Nagalakshmi U, Wang Z, Waern K, Shou C, Raha D, Gerstein M, et al. The transcriptional landscape of the yeast genome defined by RNA sequencing. Science. 2008;320(5881):1344-9. doi:10.1126/ science. 1158441.

33. Mortazavi A, Williams BA, McCue K, Schaeffer L, Wold B. Mapping and quantifying mammalian transcriptomes by RNA-Seq. Nat Methods. 2008;5(7):621-8. doi:10.1038/nmeth.1226.

34. Lister R, O'Malley RC, Tonti-Filippini J, Gregory BD, Berry CC, Millar AH, et al. Highly integrated single-base resolution maps of the epigenome in Arabidopsis. Cell. 2008;133(3):523-36. doi:10.1016/j. cell.2008.03.029.

35. Rankinen T, Zuberi A, Chagnon YC, Weisnagel SJ, Argyropoulos G, Walts B, et al. The human obesity gene map: the 2005 update. Obesity (Silver Spring). 2006;14(4):529-644. doi:10.1038/oby. 2006.71 .

36. Scherag A, Dina C, Hinney A, Vatin V, Scherag S, Vogel CI, et al. Two new Loci for body-weight regulation identified in a joint analysis of genome-wide association studies for early-onset extreme obesity in French and german study groups. PLoS Genet. 2010;6(4):e1000916. doi:10.1371/journal.pgen.1000916.

37. • Ghosh S, Dent R, Harper ME, Gorman SA, Stuart JS, McPherson R. Gene expression profiling in whole blood identifies distinct biological pathways associated with obesity. BMC Med Genomics. 2010;3: 56. doi:10.1186/1755-8794-3-56. This article demonstrates that the investigation of gene expression profiles from whole blood can inform and illustrate the biological processes related to regulation of body mass.

38. Storch KF, Lipan O, Leykin I, Viswanathan N, Davis FC, Wong WH, et al. Extensive and divergent circadian gene expression in liver and heart. Nature. 2002;417(6884):78-83. doi:10.1038/nature744.

39. Pickavance L, Tadayyon M, Williams G, Vernon RG. Lactation suppresses diurnal rhythm of serum leptin. Biochem Biophys Res Commun. 1998;248(1):196-9. doi:10.1006/bbrc.1998.8934.

40. Calvani M, Scarfone A, Granato L, Mora EV, Nanni G, Castagneto $\mathrm{M}$, et al. Restoration of adiponectin pulsatility in severely obese subjects after weight loss. Diabetes. 2004;53(4):939-47.

41. Yildiz BO, Suchard MA, Wong ML, McCann SM, Licinio J. Alterations in the dynamics of circulating ghrelin, adiponectin, and leptin in human obesity. Proc Natl Acad Sci U S A. 2004;101(28):10434-9. doi:10.1073/pnas.0403465101.
42. Tahira K, Ueno T, Fukuda N, Aoyama T, Tsunemi A, Matsumoto S, et al. Obesity alters the expression profile of clock genes in peripheral blood mononuclear cells. Arch Med Sci. 2011;7(6):933-40. doi:10. 5114/aoms.2011.26603.

43. Harris KM, Gordon-Larsen P, Chantala K, Udry JR. Longitudinal trends in race/ethnic disparities in leading health indicators from adolescence to young adulthood. Arch Pediatr Adolesc Med. 2006;160(1):74-81. doi:10.1001/archpedi.160.1.74.

44. Muoio DM, Newgard CB. Mechanisms of disease: molecular and metabolic mechanisms of insulin resistance and beta-cell failure in type 2 diabetes. Nat Rev Mol Cell Biol. 2008;9(3):193-205. doi:10. $1038 / \mathrm{nrm} 2327$

45. - Mao J, Ai J, Zhou X, Shenwu M, Ong Jr M, Blue M, et al. Transcriptomic profiles of peripheral white blood cells in type II diabetes and racial differences in expression profiles. BMC Genomics. 2011;12 Suppl 5:S12. doi:10.1186/1471-2164-12-S5-S12. This article shows different genetic markers in whole blood cells, providing valuable information about the pathophysiology of T2DM that can be used for diagnosis and pharmaceutical drug design.

46. Yvan-Charvet L, Wang N, Tall AR. Role of HDL, ABCA1, and ABCG1 transporters in cholesterol efflux and immune responses. Arterioscler Thromb Vasc Biol. 2010;30(2):139-43. doi:10.1161/ ATVBAHA.108.179283.

47. Yvan-Charvet L, Ranalletta M, Wang N, Han S, Terasaka N, Li R, et al. Combined deficiency of ABCA1 and ABCG1 promotes foam cell accumulation and accelerates atherosclerosis in mice. $\mathrm{J}$ Clin Invest. 2007;117(12):3900-8. doi:10.1172/JCI33372.

48. Yang X, Lu X, Lombes M, Rha GB, Chi YI, Guerin TM, et al. The $\mathrm{G}(0) / \mathrm{G}(1)$ switch gene 2 regulates adipose lipolysis through association with adipose triglyceride lipase. Cell Metab. 2010;11(3):194 205. doi:10.1016/j.cmet.2010.02.003.

49. Grayson BL, Wang L, Aune TM. Peripheral blood gene expression profiles in metabolic syndrome, coronary artery disease and type 2 diabetes. Genes Immun. 2011;12(5):341-51. doi:10. 1038/gene.2011.13.

50. Veenstra TD. Metabolomics: the final frontier? Genome Med. 2012;4(4):40. doi:10.1186/gm339.

51. Haider S, Pal R. Integrated analysis of transcriptomic and proteomic data. Current Genomics. 2013;14(2):20. 